\title{
Incidence of bacteraemia following fibreoptic bronchoscopy
}

\author{
M. Yigla*, I. Oren**, L. Bentur*, A. Solomonov*, N. Elias*, R. Altshuler*, A.E. Rubin*, F. Lejbkowicz ${ }^{+}$
}

Incidence of bacteraemia following fibreoptic bronchoscopy. M. Yigla, I. Oren, L. Bentur, A. Solomonov, N. Elias, R. Altshuler, A.E. Rubin, F. Lejbkowicz. (C) ERS Journals Ltd 1999. ABSTRACT: Guidelines for antibiotic prophylaxis of infective endocarditis prior to fibreoptic bronchoscopy, are based on only five studies, which showed a bacteraemia rate of $<1 \%$ among 291 patients studied. $T$ his study was designed to expand the current data regarding the frequency of bacteraemia following fibreoptic bronchoscopy.

Aerobic and anaerobic cultures of venous blood and of lavage fluid were drawn from 200 consecutive patients undergoing fibreoptic bronchoscopy without respiratory infection or antibiotic treatment prior to the procedure. The true bacteraemia rate was calculated after excluding probable "contaminated" blood cultures. A possible correlation between type of procedure performed during the bronchoscopy and occurrence of bacteraemia was investigated.

Positive blood cultures were noted following 26 bronchoscopy examinations. Coagulase negative Staphylococcus was found in the cultures of 18 patients, coagulase positive Staphylococcus in 3 patients, nonhaemolytic streptococci and a Klebsiella species in 2 patients each, and beta haemolytic streptococcus in one patient. After exclusion of 13 "contaminated" specimens the bacteraemia rate was 6.5\% (13/200 patients).

This study showed a bacteraemia rate of $6.5 \%$, significantly higher than previously recognized in a cohort of patients undergoing fibreoptic bronchoscopy without either pulmonary infection or an unusually high rate of invasive procedures.These findings should be taken into account in future evaluations of recommendations for antibiotic prophylaxis of endocarditis.

Eur Respir J 1999; 14: 789-791.
*Division of Pulmonary Medicine **Infectious Diseases Unit ${ }^{+}$Microbiology Laboratory, Rambam Medical Center and Faculty of Medicine, Technion-Israel Institute of Technology, Haifa, Israel

Correspondence: M. Yigla

Division of Pulmonary Medicine

Rambam Medical Center

POB 9602

Haifa 31096

Israel

Fax: 97248542031

Keywords: bacteraemia

fiberoptic bronchoscopy

incidence

Received: January 111999

Accepted after revision July 181999
Bacteraemia is a well-recognized invasive medical procedure. In most cases, it is a transient phenomenon without clinical consequences. In certain patients, such as those with structural cardiac abnormalities, it may lead to the development of infectious endocarditis. According to an American Heart Association statement, routine endocarditis prophylaxis is indicated prior to invasive procedures with relatively high bacteraemia rates, such as dental care, certain types of catheterization and rigid bronchoscopy [1]. Fibreoptic bronchoscopy, with or without biopsy is not included in this list due to a very low bacteraemia rate [1]. This even applies to particularly high risk patients with prosthetic valve or previous endocarditis.

A review of the literature revealed that these guidelines are based on only five studies relating to the frequency of bacteraemia following bronchoscopy, with a demonstrated overall bacteraemia rate of $0.7 \%(2 / 291$ patients $)$ [2-6]. It appears therefore, that the published guidelines for prophylactic antibiotic therapy prior to bronchoscopy are based on too few data. This led the authors to conduct a prospective study in order to expand the data on bacteraemia rates following fibreoptic bronchoscopy.

\section{Patients and methods}

The study was carried out at Rambam Medical Center, Israel, a 900 bed tertiary referral hospital. The study population consisted of 200 consecutive patients (103 hospitaliz- ed, 97 ambulatory) who underwent fibreoptic bronchoscopy during the study period. Patients with current respiratory tract infection or febrile illnesses and those receiving antibiotic therapy within a week prior to the bronchoscopy were excluded.

A total of 119 (59.5\%) patients underwent bronchoscopy for suspected malignant tumours. The remaining patients underwent bronchoscopy for investigation of: recurrent pneumonia (20 patients); haemoptysis (14 patients); stridor (13 patients); diffuse lung infiltrates (8 patients); bronchiectasis (6 patients); atelectasis, suspected foreign body and chronic cough (5 patients each); suspected upper airway stenosis and pleural effusion ( 2 patients each); and mediastinal mass (1 patient).

The procedures were performed transnasally using flexible, fibreoptic bronchoscopes (size 3.6-6 mm; Olympus America Inc., Huntington Station, NY USA). The bronchoscopy included a systematic review of the tracheobronchial tree. Endo-bronchial lesions were sampled using cup shaped (Olympus FB-19; Olympus America Inc.) or toothed (Alligator, Olympus FB-15; Olympus America Inc.) forceps (3-5 biopsy specimens), followed by 2-4 brush samples and lavage with $50-100 \mathrm{~mL}$ saline, using sterile negative pressure suction. Pulmonary lesions beyond the range of the bronchoscope were sampled using fluoroscopy-guided transbronchial biopsy and/or brushing followed by lavage. Patients with haematological conditions and bleeding disorders underwent lavage only. 
Bronchial biopsy, brushing and lavage were performed in 90 patients (45\%), brushing and lavage in $57(28.5 \%)$, and lavage in $39(19.5 \%)$. Transbronchial biopsy, brushing and lavage were performed in $11(5.5 \%)$ patients. Three patients underwent bronchoscopy solely for observation and no specimens were obtained.

\section{Bacteriological study}

Aerobic and anaerobic cultures from venous blood and from lavage fluid were drawn immediately following the bronchoscopy. Another blood culture was drawn 10-20 min later. Prebronchoscopy blood cultures from the first 100 patients were negative, excluding transient incidental bacteraemia. Blood cultures were placed in aerobic and anaerobic culture bottles. Bottles were incubated in a BacT-Alert incubator (Organon Teknica, Boxtel, the Netherlands) for a period of $\leq 5$ days at a temperature of $37^{\circ} \mathrm{C}$. Bronchial lavage fluid samples were centrifuged at $2500 \mathrm{rpm}$ at room temperature $\left(25^{\circ} \mathrm{C}\right)$ for $2 \mathrm{~min}$ and the sediment was plated on chocolate agar, MacConkey's agar, sheep blood agar, Sabouraud's agar for fungi, brain-heart infusion agar for anaerobic cultures and a selective agar for Legionella.

\section{Results}

\section{Patient characteristics}

The mean age of the patients was $54 \pm 24$ yrs (range, 6 months to 94 yrs). Twenty-nine patients $(14.5 \%)$ were children (under 18 yrs of age) and $171(85.5 \%)$ were adults. There were $152(76 \%)$ males and $48(24 \%)$ females.

\section{Past medical history, comorbidity}

Forty-eight (24\%) patients had cardiovascular disease, but none had clinical evidence of a cardiac valvular deformity; 33 (16.5\%) had a malignancy; 17 [8.5\%] had impaired immunity due to diabetes, steroid treatment, chemotherapy or radiation treatment; $16(8 \%)$ had chronic lung disease; $5(2.5 \%)$ had renal failure; $3(1.5 \%)$ had peptic disease; 2 (1\%) had collagen vascular diseases. 76 $(38 \%)$ had no defined comorbidity.

\section{Fibreoptic bronchoscopy findings}

Seventy patients had a normal study and 130 patients showed abnormalities ( 50 patients had inflamed bronchial mucosa, 49 had endobronchial lesions and 31 had signs of external pressure on major bronchi).

\section{Bacteriological findings}

Blood cultures. Twenty-six (13\%) patients had positive blood cultures following, fibreoptic bronchoscopy (table 1). Defining true bacteraemia as episodes in which two postbronchoscopy positive blood cultures yielded the same organism decreased the bacteraemia rate to $6.5 \%$ $(13 / 200)$ patients.
Table 1. - Organisms recovered from blood cultures

\begin{tabular}{lccc}
\hline & $\begin{array}{c}\text { Total } \\
\mathrm{n}\end{array}$ & $\begin{array}{c}0 \text { and } 20 \mathrm{~min} \\
\text { cultures }\end{array}$ & $\begin{array}{c}20 \mathrm{~min} \\
\text { culture+ }\end{array}$ \\
\hline $\begin{array}{l}\text { Staphylococcus coagulase } \\
\text { negative }\end{array}$ & 18 & 6 & 12 \\
$\begin{array}{l}\text { Staphylococcus coagulase } \\
\text { positive }\end{array}$ & 3 & 2 & 1 \\
$\begin{array}{l}\text { Nonhaemolytic streptococci } \\
\text { Beta haemolytic streptococci }\end{array}$ & 2 & 2 & - \\
$\begin{array}{l}\text { Klebsiella rhinoscleromatis } \\
\text { Klebsiella species }\end{array}$ & 1 & 1 & - \\
\hline
\end{tabular}

Cultures were grown from venous blood taken immediately after and $20 \mathrm{~min}$ after fibreoptic bronchoscopy. +: positive.

Lavage fluid. Cultures from lavage fluid yielded normal flora (defined as Staphylococcous coagulase negative, diptheroids and alpha haemolytic streptococci) in 120 patients and potentially pathogenic bacteria in 80 patients (one isolate in 57 patients, two isolates in 17 patients, and three isolates in 6 patients). Lavage fluid culture from 17/26 patients with positive blood cultures yielded normal flora, while the other nine lavage cultures yielded pathogenic bacteria which were different from those recovered from blood cultures.

Review of the procedures performed during the bronchoscopy in the 13 patients with true bacteraemia showed that bronchial biopsy, brushing and lavage were performed in 5 patients, brushing and lavage in 4 and lavage only in 2 patients. The remaining two patients underwent bronchoscopy solely for observation and no specimens were obtained.

Indications for fibreoptic bronchoscopy, macroscopic findings, size of bronchoscope used, and rate of invasive procedures performed during bronchoscopy did not differ significantly between the 13 patients with true bacteraemia and 187 without bacteraemia.

\section{Discussion}

This study examined the frequency of bacteraemia following fibreoptic bronchoscopy in among 200 consecutive patients. Positive blood cultures were noted following 26 bronchoscopy examinations, yielding a total bacteraemia rate of $13 \%$. True bacteraemia was recorded only for those patients in whom two consecutive blood cultures showed the same organism. This led to the exclusion of 13 bacteraemia episodes (12 Staphylococcus coagulase negative and 1 Staphylococcus coagulase positive) as contaminants. Consequently, the bacteraemia rate decreased to $6.5 \%(13 /$ 200 patients). This interpretation is supported by unpublished data showing that $70 \%$ of all staphylococcus coagulase negative blood culture isolates in the authors' institution are regarded as contaminants.

The bacteraemia rate of $6.5 \%$ shown by the current study is significantly higher than previously reported [2$6]$. The difference between the current results and those of previous studies cannot be easily explained. Nevertheless, the large cohort of patients in the study was consecutive and should be an adequate representation of the population that undergoes fibreoptic bronchoscopy. Moreover, the rate of associated invasive procedures in the study did not differ significantly between patients with or without bacteraemia 
and some bacteraemia episodes occurred even without significant mucosal injury. A study that attempts to estimate the incidence of a relatively infrequent phenomenon, such as bacteraemia following fibreoptic bronchoscopy, should include a large number of patients. The study addresses this requirement and, in fact, (to the authors' knowledge) is the first to do so.

A lack of data on upper airway flora before the bronchoscopy and the lack of correlation between the results of blood and lavage cultures cast some doubt on the significance of the findings. This might be explained by the bacteriostatic effect of lidocaine [7], used as topical anaesthesia during the bronchoscopy. Alternately, penetration of these bacteria into the blood stream might occur above the vocal cords. The isolation of Klebsiella rhinoscleromatis from the blood culture of patients with rhinoscleroma while lavage fluid was negative supports these assumptions. Still, most blood cultures revealed organisms which were also recovered from lavage fluid and are constituents of normal upper airway flora.

Bacteraemia means only the presence of viable bacteria in the blood stream. Bacteraemia may occur spontaneously or after mundane activities, such as teeth cleaning, it may complicate focal infection elsewhere or may result from surgical or dental procedures. During bronchoscopy, bacteria are driven by the bronchoscope from the upper to the lower airways. Mucosal damage induced by the bronchoscope and/or the associated procedures facilitates penetration of bacteria into the blood stream. Most bacteraemic episodes are transient, similar to that noted in the current study, with the patients remaining asymptomatic. A few patients, mostly those with cardiac structural abnormalities, might acquire infective endocarditis. To the best of the authors' knowledge, there are no data regarding the risk of acquiring infective endocarditis after fibreoptic bronchoscopy and a retrospective study could expand the data.

Fibreoptic bronchoscopy is not included in the list of procedures requiring antibiotic therapy for endocarditis prophylaxis because of an assumed low bacteraemia rate. The incidence of bacteraemia following upper and lower gastrointestinal procedures varies between $2-10 \%$ [8]. The current study has shown that the incidence of bacteraemia following fibreoptic bronchoscopy is similar to following gastrointestinal endoscopy, a procedure recognised as an indication for endocarditis prophylaxis, at least in patients with high risk cardiac conditions defined as prosthetic valve, previous endocarditis, etc. [1]
Prakash [9] recently addressed the issue of endocarditis prophylaxis prior to bronchoscopy. It was stated that the low incidence of bronchoscopy induced bacteraemia, pneumonia and systemic complications reported in previous studies does not warrant routine antibiotic prophylaxis. In the opinion of PRAKASH [9] only high-risk cardiac patients with suspected respiratory infection, in whom mucosal injury and lung damage is anticipated, should receive endocarditis prophyla xis.

The findings of this study of a $6.5 \%$ rate of bacteraemia following bronchoscopy in a cohort of patients without either pulmonary infection or a particularly high rate of invasive procedures, should be taken into account in future guidelines for endocarditis prophylaxis before bronchoscopy.

Acknowledgements. The authors thank E. Sabag for assistance in performing this study, and $\mathrm{M}$. Perlmutter for assistance in the preparation of this paper.

\section{References}

1. Dajamni AS, Taubert KA, Wilson W, et al. Prevention of bacterial endocarditis. Recommendation by the American Heart Association. JAMA 1997; 277: 1794-1801.

2. Smith RP, Sahetya GK, Baltch AL, O'hem J, Gort D. Bacteraemia associated with fiberoptic bronchoscopy. New York State J Med 1983; 83: 1045-1047.

3. Vasanthakumar V, Bhan GL, Perera BS, Taft P. A study to assess the efficacy of chemoprophylaxsis in the prevention of endoscopy-related bacteraemia in patients aged 60 and over. Quart J Med 1990; 75: 647-653.

4. Haynes J, Greenstone MA. Fiberoptic bronchoscopy and use of antibiotic prophylaxis. Brit Med J 1987; 294: 1199.

5. Pedro-Botet MI, Ruiz J, Sabria M, et al. Bacteraemia after fiberoptic bronchoscopy: prospective study. Enferm Infect Microbiol Clin 1991; 91: 159-161.

6. Kane RC, Choen MH, Fossiek BE, Tvarolzic AV. Absence of bacteraemia after fiberoptic bronchoscopy. $\mathrm{Am}$ Rev Res Dis 1975; 111: 102-104.

7. De Clare F. Antimicrobial activity of local anaesthetics. Intensive Care Med 1992; 18: 380.

8. Shorvon PJ, Eykyn SJ, Cotton PB. Gastrointestinal instrumentation, bacteraemia, and endocarditis. Gut 193; 24: 1078-1093.

9. Prakash UBS. Prophylactic antibacterial therapy for bronchoscopy: Indications. J Bronchol 1997; 4: 281-285. 